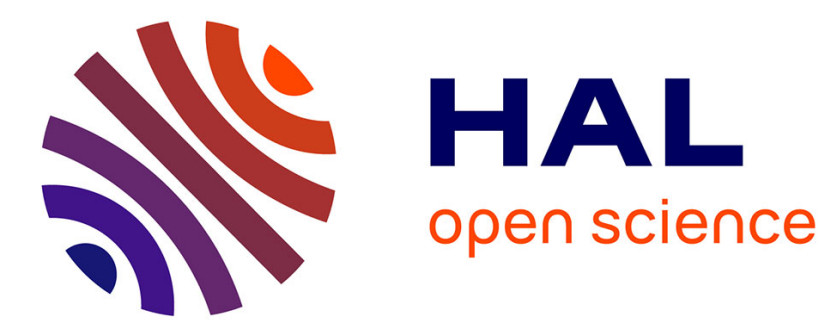

\title{
Du capital culturel aux patrimoines culturels
}

\author{
Christian Barrère, Cyril Hédoin
}

\section{To cite this version:}

Christian Barrère, Cyril Hédoin. Du capital culturel aux patrimoines culturels. Revue d'économie régionale et urbaine, 2014, 10.3917/reru.145.0801 . hal-02569033

\section{HAL Id: hal-02569033 \\ https://hal.science/hal-02569033}

Submitted on 10 May 2020

HAL is a multi-disciplinary open access archive for the deposit and dissemination of scientific research documents, whether they are published or not. The documents may come from teaching and research institutions in France or abroad, or from public or private research centers.
L'archive ouverte pluridisciplinaire HAL, est destinée au dépôt et à la diffusion de documents scientifiques de niveau recherche, publiés ou non, émanant des établissements d'enseignement et de recherche français ou étrangers, des laboratoires publics ou privés. 


\section{Christian Barrère Cyril Hédoin}

Université de Reims Champagne Ardenne

\section{Du capital culturel aux patrimoines culturels}

\section{in \\ Revue d'Économie Régionale \& Urbaine}

2014/5 (décembre), pp. 801-824

$$
\text { DOI : 10.3917/reru.145.0801. URL : https:// }
$$
www.cairn.info/revue-d-economie-regionale-eturbaine-2014-5-page-801.htm 


\title{
Du capital culturel aux patrimoines culturels
}

\section{From cultural capital to cultural heritages}

\author{
Christian BARRÈRE \\ Laboratoire REGARDS (Université de Reims) et ISMEA (Paris) \\ christian.barrere@gmail.com

\section{Cyril HÉdOIN} \\ Laboratoire REGARDS (Université de Reims) \\ cyril.hedoin@univ-reims.fr
}

Mots-clés : capital culturel, créativité, cultural commons, patrimoines culturels

Keywords : creativity, cultural capital, cultural commons, cultural heritages

Classification JEL : B52, Z10 


\section{Résumé}

L'objet de l'article est d'étudier la façon dont l'analyse économique prend en compte la dimension temporelle des ressources et produits culturels à l'heure où, dans le cadre d'économies de la connaissance, la culture a un rôle économique croissant. Si BECKER (1964) raisonne en termes de capital culturel, d'autres économistes insistent sur les propriétés créatives du capital culturel dans une approche en termes de créativité. Les limites de l'identification des ressources culturelles à un capital nous conduisent à nous intéresser aux modes de gestion des ressources culturelles par des institutions communes en appliquant l'analyse des commons au champ culturel. Néanmoins cette approche n'est pas suffisante pour traiter de ressources culturelles particulières en ce que, loin de constituer de simples agrégats de ressources, elles présentent un caractère structuré et traversent le temps. La dernière partie propose des pistes pour dépasser les limites précédentes au moyen d'une approche patrimoniale.

\section{Abstract}

This article studies how economics takes into account the time dimension of cultural resources and products in the context of the economy of knowledge, where culture has an increasing economic importance. While BECKER (1964) conceives culture as a capital, other economists rather take a creativity approach and emphasize the creative properties of cultural capital. However, the assimilation of cultural resources to some kind of capital is problematic. This leads us to study the regulation of cultural resources on the basis of institutions through the application of the commons analysis to the cultural realm. However, this approach is insufficient to analyze some specific cultural resources that, instead of being simple aggregates of resources, have structural and temporal properties. The last section surveys several ways to overtake these limits through an approach in terms of heritage.

L'économie de la culture est aujourd'hui reconnue comme une branche des sciences économiques et les politiques culturelles tiennent de plus en plus compte de leurs conditions et conséquences économiques. C'est dire que la culture n'apparaît plus comme un champ autonome, entièrement extérieur à celui du marché, et particulièrement comme un moyen d'échapper à la logique utilitariste de la rationalité instrumentale, mais comme un domaine particulier de l'économie marchande. En même temps la culture peut-elle être purement et simplement assimilée à une marchandise comme une autre, bien de consommation standard ou input des combinaisons productives? Et, notamment, comment tenir compte de la durée de vie exceptionnelle de certains éléments culturels, qu'il s'agisse des langues qui se transmettent durant des siècles ou de certaines œuvres qui peuvent être conservées pendant des millénaires en échappant à toute obsolescence ? L'objet de cet article est précisément d'interroger l'analyse économique sur son mode de traitement du caractère temporel des ressources culturelles. Nous cherchons à montrer que la prise en compte des dimensions économiques de la culture, en la transformant en capital culturel, ne permet pas d'intégrer des caractéristiques économiques essentielles de celle-ci. Nous proposons alors un mode alternatif de conceptualisation des ressources culturelles qui traversent le temps en constituant des patrimoines, au moyen d'une approche patrimoniale. 


\section{-1 - \\ De la marchandisation de la culture aux patrimoines culturels}

Le premier lien établi entre culture, marché et capital ne date pas de BECKER et de sa théorie du capital humain (BECKER, 1964) mais de l'approche marxiste critique de l'École de Francfort. Celle-ci, dès la première moitié du Xx ${ }^{e}$ siècle, interprète les changements fondamentaux qui se produisent dans le domaine culturel comme la naissance d'une culture de masse adaptée à une société de masse (HORKHEIMER et ADORNO, 1947 ; BENJAMIN, 1936). Des développements technologiques permettent la production, la reproduction et la distribution de masse des produits culturels, dont la Factory d'Andy WARHOL constitue de ce point de vue un symbole parfait, créant une rupture avec le modèle précédent basé sur la singularité et l'unicité de l'œuvre artistique. La culture entre alors dans l'ère de la marchandise et fonctionne selon un schéma industrialiste jadis caractéristique des seuls biens industriels (production avec des équipements, supposant des investissements financiers élevés et permettant des économies d'échelle...). Ce processus conduit à une soumission du domaine de la culture à la régulation économique marchande. Les biens artistiques perdent leur aura (BENJAMIN, 1936), leur dimension religieuse et spirituelle. Ils deviennent abordables et entrent dans la sphère de la consommation et de la communication de masse. À partir de ce moment, la culture de masse se transforme en industrie culturelle et sert à la domination des masses. L'industrialisation permet de produire et de vendre des produits culturels à coût réduit et de les mettre ainsi à la disposition de la plupart des consommateurs alors que leur usage avait été jusque-là limité à des groupes sociaux déterminés. La révolution du livre de poche est significative de ce processus. La révolution numérique $\mathrm{a}$, par la suite, décuplé les possibilités de produire en masse des biens culturels nouveaux et différenciés, en particulier dans le domaine du multimédia. La mondialisation a accru la taille des marchés potentiels comme le révèlent les chiffres de ventes des albums de pop music et des best-sellers ou les recettes de certains films.

BENJAMIN (1936) et l'École de Francfort se référaient aux produits culturels traditionnels directement liés au champ artistique : les livres et la littérature, la peinture, le cinéma et le théâtre. Deux nouveaux changements se sont joints à ce dernier. Le premier est le développement de la production et de la consommation de biens et services porteurs de caractéristiques sémiotiques (BARRÈRE et SANTAGATA, 1998) qui se réfèrent aux goûts et au plaisir plus qu'à la raison et la nécessité (BARRÈRE, 2013) et rejoignent le champ toujours croissant des biens culturels. Ces « biens de goût » incluent la mode, le design, la gastronomie, la production de vins et alcools, le tourisme et d'autres activités similaires (LIPOVETSKY, 1987, 2006 ; ROCHEFORT, 2001) et contribuent de plus en plus à la croissance économique. Les dépenses en matière de tourisme représentent aujourd'hui $9 \%$ du produit mondial et 250 millions d'emplois, ce qui en fait la première industrie au monde ; les industries du luxe ont un chiffre d'affaires qui dépasse les 200 milliards d'euros. En outre, de plus en plus de produits, même s'ils ont une fonction principalement utilitaire, intègrent des caractéristiques culturelles : les automobiles incorporent de plus en plus de design, 
les producteurs d'agroalimentaire industriel dépensent de plus en plus d'argent pour emballer, présenter leurs produits et leur donner une image attirante.

Le second grand changement est l'utilisation accrue de la culture dans le processus productif. La production dépend de plus en plus étroitement de la technologie et de la science mais aussi de la créativité et des arts (LANDRY et BIANCHINI, 1995 ; DCMS, 2001 ; FLORIDA, 2002 ; CAVES, 2000 ; TOWSE, 2003). Le triomphe des biens créés par Apple illustre parfaitement cette combinaison de créativité technologique et de créativité artistique dans la conception de nouveaux produits et de nouveaux designs. Alors que l'École de Francfort s'intéressait à la culture comme nouveau débouché de la production capitaliste, les théories de la créativité et de la société de connaissance mettent l'accent sur sa dimension productive.

Si la culture passe de plus en plus par le marché, la relation culture-marché présente des particularités du point de vue de l'économiste. En premier lieu, la culture apparaît à la fois comme domaine de consommation individuelle (l'audition d'un CD) et aussi comme input individuel, par exemple via l'élévation des connaissances et compétences individuelles, mais aussi comme domaine de consommation collective (la fréquentation des musées) et comme input collectif, la culture d'une époque permettant de créer de nouvelles œuvres ou de nouvelles aménités à partir d'œuvres existantes. En second lieu, des biens culturels, comme les monuments ou œuvres célèbres, échappent à l'usure du temps et leur valeur peut même s'accroître comme dans le cas des tableaux. Certains développent des effets externes, de type spill-over, et sont à la source de nouvelles utilités. Des biens culturels fonctionnent comme capital et/ou comme patrimoine, n'étant souvent pas gérés par la seule logique marchande, voire apparaissant comme commons. Ils nous intéressent particulièrement parce que leur rôle, souvent négligé, commence à être appréhendé et s'avère souvent déterminant. Le modèle de la Troisième Italie (BECCATINI, 2004) se fonde sur le rôle du patrimoine culturel interprété comme un ensemble d'actifs spécifiques, individuels, collectifs et sociaux, qui permettent la mise en place de formes de coopération entre acteurs ainsi que de fortes incitations à l'innovation. L'UNESCO, devant la constatation de la disparition ou de la dégradation de patrimoines (chaque année, selon elle, plus d'une centaine de langues disparaissent dans le monde), étend sa protection au patrimoine culturel intangible. Dans le même temps, des compétences en matière de travail artisanal, et aujourd'hui industriel, sont perdues, mais d'autres patrimoines, en revanche, prennent une plus grande valeur et sont l'objet de gestions sophistiquées. Des acteurs privés " patrimonialisent », c'est-à-dire constituent en patrimoines des actifs privés ou publics, voire des institutions. Les nouveaux groupes du luxe, comme LVMH et KERING achètent des entreprises de haute couture, de prêt-à-porter, de luxe, pour mettre en valeur leur capital de réputation et leur patrimoine de savoir-faire.

La théorie économique a donc développé diverses interprétations de cette nouvelle relation entre culture et économie et, plus précisément, de cette dimension temporelle caractéristique de la culture comme ressource économique. Nous examinerons dans une première partie les approches qui lient ressources culturelles et production de valeur via la notion de capital. Si BECKER (1964) raisonne en termes de capital 
culturel (2.1), d'autres économistes insistent sur les propriétés créatives du capital culturel (LANDRY et BIANCHINI, 1995 ; DCMS, 2001 ; FLORIDA, 2002 ; POTTS, 2012), et substituent à l'approche traditionnelle de la fonction de production une approche en termes de créativité (2.2). Les limites de l'identification des ressources culturelles à un capital (3.1) nous conduiront à nous intéresser aux modes de gestion des ressources culturelles par des institutions communes (OSTROM, 1990, 2006) en appliquant l'analyse des commons au champ culturel (Hess et OSTROM, 2003 ; MADISON et al., 2010 ; BERTACCHINI et al., 2012), ce qui permet de prendre explicitement en compte le caractère collectif de la culture et d'analyser l'imbrication des formes marchandes et non-marchandes, individuelles et collectives, qui organisent son rôle économique (3.2). Néanmoins, cette approche n'est pas suffisante pour traiter de ressources culturelles particulières en ce que, loin de constituer de simples agrégats de ressources, elles présentent un caractère structuré. Il existe en effet, au sein de l'ensemble des ressources culturelles traversant le temps, des sous-ensembles relativement cohérents et structurés qui peuvent être analysés comme des patrimoines, sur le modèle des patrimoines que constituent les monuments historiques ou de ceux que protège l'UNESCO, et qui ont aujourd'hui un rôle décisif pour la vie sociale mais aussi économique via les industries créatives. La dernière partie (4) propose alors des pistes pour dépasser les limites précédentes au moyen d'une approche patrimoniale.

\section{- 2 - \\ Les ressources culturelles comme capital}

\subsection{Les ressources culturelles comme capital culturel}

La relation entre la culture et l'économie, et plus particulièrement entre valeur culturelle et valeur économique, constitue un objet d'étude pour les économistes depuis maintenant plusieurs décennies. BECKER (1964) est l'auteur d'une contribution précoce et significative à cette problématique par son traitement de la culture au travers du concept de capital humain. Le capital humain correspond au stock de compétences, de connaissances et d'expériences possédé par chaque individu et qui induit un accroissement de leur productivité. Dans l'analyse proposée par BECKER (1964), la culture relève du capital humain des agents en tant qu'ensemble d'expériences et est donc de ce point de vue considérée comme un élément contribuant à l'efficience productive.

Récemment, BECKER (1996) a étendu son analyse à la problématique de la formation des préférences. Dans le cadre d'analyse qu'il développe, les préférences des agents sont déterminées par deux stocks de capital, le capital personnel (P) et le capital social (S). Le capital personnel et le capital social sont deux éléments constitutifs du capital humain de chaque individu. Formellement, ces deux types de capital sont intégrés dans une fonction d'utilité étendue qui incorpore, en plus du traditionnel panier de biens $\mathrm{B}$ consommé par l'agent à un moment $t$, les deux stocks de capital. Ainsi, au temps $t$, l'utilité d'un agent $i$ est déterminée par la fonction suivante : 
$\mathrm{u}_{\mathrm{it}}=\mathrm{u}_{\mathrm{it}}\left(\mathrm{B}_{\mathrm{it}}, \mathrm{P}_{\mathrm{it}}, \mathrm{S}_{\mathrm{it}}\right)$

Cette fonction d'utilité étendue est supposée être stable dans le temps. Cependant, étant donné que les choix présents peuvent affecter le niveau futur des stocks de capital personnel et social, le même choix peut générer des niveaux d'utilité différents en fonction du moment où il est effectué. Par conséquent, tandis que les préférences étendues déterminées par le capital personnel et social sont stables et données, la fonction d'utilité décrivant uniquement les préférences sur les biens de consommation est instable et peut changer en fonction de l'investissement en capital personnel ou social de l'agent.

BECKER (1996) définit le capital personnel comme l'ensemble des consommations passées et des expériences personnelles affectant les niveaux présents et futurs d'utilité. Ce capital est affecté d'un " taux de dépréciation psychologique » $d_{p}$ et peut être accru d'un montant $x_{t}$ par le biais du comportement et des choix présents. Par conséquent, le stock de capital personnel de la période suivante $t+1$ est égal à la formation de capital personnel à la période présente $x_{t}$ à laquelle s'ajoute la valeur présente actualisée du stock de capital personnel :

$P_{i t+1}=x_{i t}+\left(1-d_{p}\right) P_{i t}$

Le même raisonnement s'applique dans le cas du capital social. Le capital social inclut de manière générale tous les facteurs susceptibles d'affecter les niveaux présent et futur d'utilité d'un agent au travers de ses interactions sociales : "Men and women want respect, recognition, prestige, acceptance, and power from their family, friends, peers, and others. Consumption and other activities have a major social component partly because they take place in public. As a result, people often choose restaurants, neighborhoods, schools, books to read, political opinions, food, or leisure activities with an eye to pleasing peers and others in social network » (BECKER, 1996). Il y a toutefois une différence significative qui vient du fait que le niveau de capital social d'un agent $i$ ne dépend pas exclusivement des choix de ce dernier. En effet, la nature même du capital social implique que son niveau est une fonction des choix faits par les membres du groupe ou de la communauté d'appartenance de l'individu. De ce fait, le niveau présent $X_{i t}$ de capital social d'un agent $i$ au moment $t$ est une fonction de la consommation de biens sociaux par tous les agents appartenant au réseau de relations sociales de l'agent $i$. Dans la formalisation proposée par BECKER (1996), $X_{i t}$ est défini comme la somme de la consommation de biens sociaux par tous les agents $j$ qui appartiennent au réseau de $i$. Autrement dit, $X_{i t}=\sum_{j} x_{j t}$. Dans la même manière que pour le capital personnel, un taux de dépréciation $d_{s}$ est appliqué au capital social. Par conséquent, la valeur $S_{t+1}$ du stock de capital social en $t+1$ se forme de la manière suivante :

$\mathrm{S}_{\mathrm{it}+1}=\mathrm{X}_{\mathrm{it}}+\left(1-\mathrm{d}_{\mathrm{s}}\right) \mathrm{S}_{\mathrm{it}}$

Une autre différence importante entre les deux types de capitaux réside dans le fait que les agents ont un contrôle très limité sur le niveau de capital social. En effet, il apparaît clairement que l'effet marginal des décisions d'un agent sur le niveau de son stock de capital social va en décroissant avec la taille du réseau auquel il appartient. 
Un autre aspect notable du capital social est sa propension à générer des régularités comportementales cumulatives et auto-renforçantes. Le stock de capital social, ainsi que l'investissement dans ce type de capital, sont en effet généralement fortement complémentaires en raison du fait que l'utilité marginale tirée de la consommation des biens sociaux tend à s'accroître avec le niveau présent du stock. Par exemple, une fois qu'un agent est devenu membre d'un gang, l'utilité marginale des activités criminelles augmente parce que ces dernières contribuent à renforcer la réputation de l'agent.

BECKER (1996) appréhende la culture comme une forme particulière de capital social. En tant qu'ensemble de "valeurs partagées et de préférences transmises de génération à génération, au travers de la famille, des pairs, des groupes ethniques, des classes et d'autres groupes ", la culture contribue à déterminer les coûts et l'utilité des activités et de la consommation des différents biens. La spécificité de la culture réside dans le fait qu'elle change lentement et qu'elle est quasiment insensible aux actions des individus. Par conséquent, la relation entre l'action des agents et la culture est, au moins sur le plan idéal typique, unilatérale : les individus n'ont pas la capacité de changer la culture, mais la culture a une influence significative sur le comportement des individus. Selon BECKER, cette caractéristique rend beaucoup plus pertinente la traditionnelle hypothèse faite par les économistes concernant la stabilité et l'exogénéité des préférences sur les biens, que dans le cas du capital personnel ou même des autres formes de capital social.

Au-delà de ces éléments, l'analyse de BECKER (1996) de la culture comme capital social est peu informative sur la manière dont la culture construit et oriente l'action individuelle. Par ailleurs, l'assimilation de la culture à une forme de capital humain n'offre pas d'explication concernant la formation de la valeur culturelle, et la relation de cette dernière avec la valeur économique. Cette question est traitée par THROSBY (1999) dans une tentative de dépassement de ce cadre d'analyse. D'après THrosby (1999), le capital culturel doit être distingué à la fois de la notion de capital physique, mais aussi des notions de capital humain et de capital naturel, telles qu'elles sont traditionnellement appréhendées par les économistes. THROsBY (1999) distingue ainsi le sens fonctionnel et le sens constitutif de la culture. Dans son sens fonctionnel, la culture renvoie à toutes les activités économiques qui prennent place au sein des « industries culturelles » ou du secteur culturel d'une économie. Dans son sens constitutif, la culture correspond à l'ensemble d'attitudes, de pratiques et de croyances qui sont à la base du fonctionnement de la société. Dans ce sens général, « something can be said to be of cultural value if it contributes to these shared elements of human experience " (THROsBY, 1999). Si l'on met de côté les difficultés relatives à la mesure de la valeur culturelle, un item relevant du capital culturel se définit comme un actif contribuant à la formation de la valeur culturelle: "cultural capital is the stock of cultural value embodied in an asset" (THROSBY, 1999).

Les actifs culturels peuvent être tangibles (bâtiments, structures, artefacts culturels tels que des peinture ou des sculptures, etc.) ou intangibles (idées, pratiques, croyances, valeurs, traditions, biens relevant du domaine public comme la littérature ou la musique). Dans la plupart des cas, les actifs culturels créent de la valeur 
économique et/ou culturelle supplémentaire. En ce qui concerne les biens tangibles, la valeur économique peut tout simplement provenir de leur existence physique. Mais elle peut aussi provenir de ou être augmentée par leur valeur culturelle, dérivée de certaines de leurs caractéristiques. Ainsi, la valeur économique d'une peinture de VAN GOGH telle que mesurée par son prix sur le marché de l'art est bien supérieure à sa valeur " physique » en raison de son contenu culturel. Il en va différemment pour les actifs culturels intangibles car ils n'ont pas de valeur économique directe. Mais un stock d'actifs culturels intangibles va générer un flux de services lui-même porteur de valeur économique et culturelle. Un corollaire est que l'ordre de préférences sur les actifs culturels ne sera pas nécessairement le même, suivant que l'on s'attache à leur valeur culturelle ou à leur valeur économique. Enfin, une importante implication est que le capital culturel d'une communauté permet d'accroître de manière significative la valeur économique de sa production, ce qui indique que le capital culturel est une composante à part entière de la fonction de production de cette communauté.

Toutefois, même si l'analyse de Throsby (1999) identifie la culture comme un capital spécifique ayant une importance économique propre, les approches en termes de capital culturel restent insatisfaisantes, ainsi que nous l'indiquons plus bas. L'identification de la culture à un capital, au sens où l'entend l'économiste, est problématique car elle revient à conférer aux ressources culturelles des propriétés qui ne sont pas les leurs, et au contraire à en ignorer d'autres qui leur sont spécifiques. On peut, par exemple, noter que l'hypothèse posée par BECKER (1996) de dépréciation du capital (personnel et social) est particulièrement discutable dans le cas des biens culturels. La valeur culturelle d'une peinture ou d'une œuvre littéraire, sur laquelle repose une large partie (ou la totalité) de sa valeur économique, n'est ainsi pas soumise à une dépréciation dans le temps. Au contraire, la consommation de ce type de bien est susceptible d'accroître sa valeur culturelle et économique, au travers de la formation d'un patrimoine pouvant prendre la forme, par exemple, d'échanges et d'analyses autour de l'œuvre permettant de mieux en apprécier le contenu. De manière plus générale, le rapport au temps des ressources culturelles est très différent de celui des autres formes de capital. Outre leur caractère le plus souvent non-rival sur le plan inter-temporel, les biens culturels donnent généralement lieu à un processus d'accumulation au regard des expériences de consommation et de production. Ces éléments, ainsi que d'autres, suffisent à établir la nécessité d'un dépassement de l'approche économique de la culture en termes de capital. Un premier dépassement concerne le rôle productif du capital avec le développement des approches en termes de créativité.

\subsection{Les ressources culturelles comme capital créatif}

Au tournant du siècle, des économistes ont proposé une nouvelle approche pour comprendre les relations entre économie et culture, et principalement les effets économiques de la culture. Ils tiennent compte de la dimension temporelle de la culture en montrant comment la culture peut créer sa propre extension et donc, au lieu de raisonner en termes de capital culturel, le font en termes de créativité. 


\subsubsection{Définitions}

Le nouveau champ au sein duquel sont étudiées les conséquences économiques de la culture est celui des industries créatives ou Creative Industries (CI). Cependant, aucune définition de celles-ci n'est universellement admise. CAVES (2000) définit les industries créatives comme les industries " in which the product or service contains a substantial element of artistic or creative endeavour » et a une valeur culturelle, artistique ou de loisir. TOWSE (2003) identifie industries culturelles et industries créatives : "Nowadays also called "creative industries", cultural industries mass-produce goods and services with sufficient artistic content to be considered creative and culturally significant. The essential features are industrial-scale production combined with cultural content". Throsby (2001) décrit les industries culturelles (assimilées aux industries créatives ou également définies comme industries à copyright) par un modèle de cercles concentriques, selon le degré de créativité qu'elles incorporent.

Le département pour la culture, les medias et les sports (DCMS) du gouvernement britannique, à la pointe dans l'étude de ces industries et dans la définition de politiques les concernant, les définit comme " those industries which have their origin in individual creativity, skill and talent and which have a potential for wealth and job creation through the generation and exploitation of intellectual property (DCMS, 2001). Une étude importante faite pour la Commission européenne prend pour objet le « cultural \& creative sector ". À côté du secteur culturel, "In the "creative sector", culture becomes a "creative" input in the production of non-cultural goods. It includes activities such as design (fashion design, interior design, and product design), architecture, and advertising. Creativity is understood in the study as the use of cultural resources as an intermediate consumption in the production process of non-cultural sectors, and thereby as a source of innovation » (KEA, 2006).

Selon le ministère des Affaires étrangères français, les industries culturelles et créatives sont caractérisées par leur dualité culturelle et économique. Elles incluent: les activités culturelles archétypiques ou activités non industrielles qui produisent des prototypes protégés par des droits de propriété comme les arts visuels (peinture, sculpture, photographie, artisanat), les arts du spectacle et le patrimoine (musées, bibliothèques, sites archéologiques et historiques, archives), les industries culturelles (film et vidéo, télévision et radio, jeux vidéos, musique, édition), les industries créatives, industrielles ou de prototype (design, architecture, publicité, industries du goût -gastronomie, vins et spiritueux -, industries du luxe - mode, parfumerie tourisme, sports, industries éducatives, les industries de support et de communication (Web numérique, ordinateurs, baladeurs, etc.).

La difficulté à définir le périmètre des Ci traduit la présence, à doses variées, de la créativité dans un grand nombre d'industries, mais manifeste aussi le mixage qui s'est établi entre créativité et production de masse. Nous pouvons alors caractériser les industries créatives par:

- l'utilisation de la production de masse, même si ces secteurs combinent souvent des processus de production hétérogènes, manuels, néo-artisanaux (production en série avec des équipements spécifiques et l'intervention de travailleurs), industriels ou 
encore des procès de production unissant productions matérielles et immatérielles ; - l'intégration de la créativité comme input important ou dominant.

\subsubsection{Un nouveau paradigme productif}

L'approche des Ci remet en cause l'approche du capital culturel en proposant une nouvelle interprétation de la culture comme moyen de production. Cette dernière s'inscrit en effet dans l'approche standard de la fonction de production qui implique que la production résulte de ressources précises, les inputs, parmi lesquels le capital, et que la relation entre inputs et outputs puisse être établie sans équivoque comme le postule l'analyse de la productivité marginale. Cela implique de nombreuses conditions implicites, généralement admissibles au prix de simplifications et de conventions dans le cas d'une production industrielle classique. Les inputs doivent être : identifiables, séparables, homogènes, reproductibles, mesurables, et organisés en combinaisons connues et stables. La spécificité de la création est analysée par l'image de la création divine; Dieu crée le monde parce qu'il le fait à partir de rien. De la même façon, la création de l'artiste ou du styliste de mode est faite à partir de rien, hors de tout recours à une ressource économique standard, mais grâce à une ressource singulière, qu'on l'appelle génie, créativité, inspiration, ou illumination, ressource qu'on ne peut analyser comme un input classique parce que son fonctionnement est idiosyncrasique et dépend de très nombreux facteurs. Les mêmes musiciens n'atteignent pas la même qualité s'ils sont dirigés par KARAJAN ou par un chef ordinaire. L'output est souvent loin de toute mesure raisonnable (d'autant qu'il a souvent des effets directs et individualisables mais aussi des effets indirects ou collectifs) et la relation input-output ne peut s'exprimer par un ratio. De plus, le résultat du processus créatif varie avec l'environnement ou même de façon aléatoire. Comme on est dans une économie de variété infinie (CAVES, 2000) les inputs créatifs $\mathrm{n}$ 'interviennent pas selon le schéma habituel et la relation entre ressources et valeur de leurs effets est souvent indéterminée. Les mêmes inputs donnent parfois une œuvre géniale et parfois un résultat médiocre. Les combinaisons productives ne peuvent être considérées comme connues et stables, alors que l'idée de création se réfère à l'idée d'une production à partir de rien, rien d'identifiable précisément en tout cas. Quand on demandait à Yves SAINT LAURENT comment il travaillait, il racontait qu'il ne se donnait pas de programme précis, qu'il s'installait à son bureau et rêvassait, et que, parfois, tout d'un coup, une idée lui venait, qu'il se mettait alors à dessiner, et qu'ensuite il mettait à la corbeille toute une partie de ses esquisses pour n'en garder que quelques-unes, ensuite retravaillées. Tel autre grand couturier dit passer ses soirées en boîte de nuit et, parfois, à son retour, avoir " un flash ", convoquer alors en pleine nuit ses assistants des quatre coins de Paris, leur raconter ses visions, et les inviter à concrétiser l'idée du maître pendant que lui rentre se coucher.

Dans le domaine de la création, les outils économiques standards n'expliquent rien : établir une fonction de production pour les tableaux de PICASso ou les robes de LAGERFELD n'a guère de sens. Le principe de mesure du temps et d'économie de temps, dans les phases de création proprement dite, perd tout sens. En quoi cela importe-t-il de savoir si PICASSO a peint ce tableau en un jour ou une semaine, HAENDEL composé le Messie en un mois ou un an ? Un cas classique dans l'histoire de la musique est d'opposer la Messe en si mineur de BACH et le Messie de HAENDEL, 
composés à la même époque. Alors que BACH a mis vingt-six ans à composer sa Messe, réutilisant des créations antérieures, selon le mode habituel de la parodie, créations qui résultaient elles-mêmes d'un long travail, HAENDEL écrivit son Messie en vingt et un jours. Personne ne songerait à indexer la qualité relative des deux œuvres sur le temps consacré à leur écriture !

L'approche de la créativité remet en cause le paradigme de la production des XIX et $\mathrm{Xx}^{\mathrm{e}}$ siècles - un paradigme énergétiste de la production - commun à l'approche classico-marxienne et à l'approche walrasienne, et selon lequel la richesse est accumulation de marchandises, biens reproductibles produits avec du travail, direct et indirect. Pour produire, il suffit de consacrer à la production les dépenses nécessaires, en prix ou en quantité de travail. On produit en transformant de l'énergie (humaine ou naturelle) en marchandises selon une logique énergétiste, conforme à la grande vision du rapport homme-nature de ces siècles. Les biens "rares ", c'est-à-dire non reproductibles avec du travail humain, sont des biens marginaux et disparaissent donc de l'analyse comme ils semblent disparaître du cœur de l'économie.

Un tel paradigme interdit de penser la création autrement que comme quelque chose de marginal (réduit notamment au domaine de l'art, expulsé de celui de la production "normale »). L'introduction dans l'analyse économique d'un paradigme de l'information a constitué une première avancée. Les discussions tenues aujourd'hui autour de la créativité cherchent à aller au-delà.

\subsubsection{Connaissance cumulative et connaissance non-cumulative : le rôle des patrimoines}

STEINER (2001) a fait remarquer que, alors que le progrès scientifique et technique était cumulatif, les œuvres les plus anciennes en musique ou en littérature n'ont jamais été dépassées : " major art is not relegated to antiquarian status; Chartres does not date... What... is in advance on Homer or Sophocles, on Plato or Dante? Beyond Hamlet, what novel surpasses Madame Bovary or Moby Dick?"

La science est le modèle des domaines mettant en œuvre une créativité qui prend la forme de la connaissance cumulative. Les scientifiques se servent comme inputs de l'ancienne connaissance scientifique pour produire de nouvelles connaissances, plus développées, plus rigoureuses, plus sophistiquées, de telle sorte que la connaissance ancienne se fond dans la nouvelle et cesse d'avoir une valeur en tant que telle. $\mathrm{Au}$ contraire, comme le note STEINER, il y a des produits de la créativité noncumulatifs quand la production de certains biens créatifs déterminés n'améliore aucune connaissance préalable et n'a pas vocation à être remplacée dans le futur. Cela explique pourquoi la définition des industries culturelles et créatives par le ministère des Affaires étrangères français inclut le patrimoine : musées, bibliothèques, sites archéologiques et historiques, archives, etc. Les patrimoines jouent un grand rôle dans les arts et les industries créatives telles la mode, les vins et spiritueux, la gastronomie. Pour présenter autrement les choses, on peut dire que dans les champs gouvernés par la connaissance cumulative, les patrimoines sont fongibles alors que, dans ceux qui sont basés sur la connaissance non-cumulative, ils ne le sont pas. Ainsi, s'intéresser aux industries créatives implique d'introduire le rôle clef 
des patrimoines. Les patrimoines sont les compléments de la créativité ; ce sont des stocks formés par la créativité du passé et ils peuvent servir pour développer une nouvelle créativité. La culture intervient ainsi comme " nouvelle » culture, la culture est alors un flux, via la créativité, et comme " ancienne » culture, la culture est un stock, via le patrimoine. La Figure 1 représente les industries créatives sur la base du schéma classique de THRosby mais en incluant le rôle des patrimoines et en distinguant entre connaissance cumulative et non cumulative.

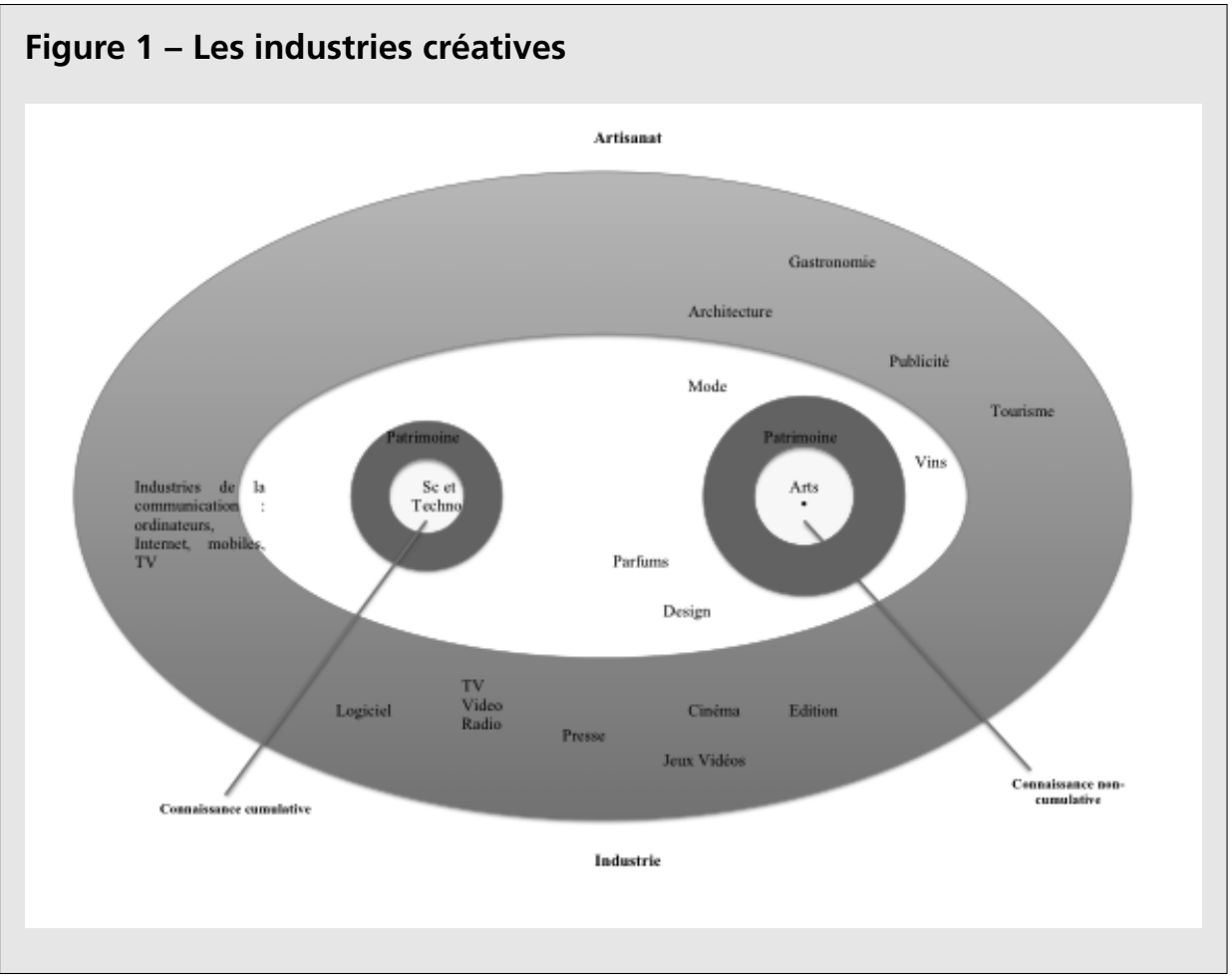

Nous représentons les industries créatives par une ellipse dont les deux foyers correspondent aux deux sources de caractéristiques productives de ces industries, la connaissance cumulative et la connaissance non cumulative. Les industries recourent plus ou moins à chacune d'entre elles. Autour du premier foyer (connaissance cumulative), nous figurons par un premier cercle la science et la technologie, qui sont les lieux principaux de développement de cette connaissance, par un second le patrimoine qu'elles secrètent. Ce cercle est petit parce qu'il s'agit de patrimoines fongibles, qui disparaissent en grande partie. Autour du second foyer (connaissance non-cumulative), le premier cercle correspond aux arts, lieu essentiel de développement de ce type de créativité, et le second, d'ampleur plus grande que dans le cas du foyer précédent, aux patrimoines de type artistique. Ils forment pour longtemps des sources essentielles d'utilités et de créativité. Le terme « patrimoine » est utilisé dans son sens large et ne se réfère pas seulement, comme dans les classifications 
officielles françaises (et souvent étrangères), aux musées, bibliothèques... mais à tous les patrimoines constitués par l'accumulation et la sédimentation de la créativité, comme le patrimoine de savoir-faire des maisons de couture ou les recettes de cuisine caractéristiques des cultures culinaires. Les autres secteurs créatifs sont localisés dans l'ellipse selon deux critères : leur distance de chaque foyer, leur relation à un processus de production plutôt industriel ou plutôt artisanal.

L'approche des industries créatives tend à considérer la culture, non pas comme un capital traditionnel, mais comme une source de créativité, qui apparaît comme créativité nouvelle et comme créativité accumulée sous la forme de patrimoines. Une seconde caractéristique importante de l'approche est que la création est reliée à son contexte social, historique et spatial. L'analyse marshallienne des districts industriels avait déjà montré que certains lieux étaient, à certains moments, et dans certains contextes, capables de développer une atmosphère spécifique qui contribuait à étendre les effets de spill-over et à accroître la créativité. FLORIDA (2002) en étudiant les villes créatives et SANTAGATA (2002), ZuKIN et BRASLOW (2011) les districts culturels et créatifs développent ce type d'analyse pour d'autres configurations que les districts industriels traditionnels. L'hétérogénéité des territoires contribue à former des actifs stratégiques. Dès lors, les patrimoines, comme nous l'avons fait, peuvent être introduits. Leur analyse nécessite aussi celle des formes sociales qu'ils prennent, qu'elles soient publiques ou privées, qu'ils fonctionnent comme capital ou non. Leur détention n'est pas toujours privée, comme quand l'UNESCO établit la liste représentative du patrimoine culturel intangible de l'humanité.

\section{- 3 - \\ Des limites du capital culturel aux cultural commons}

\subsection{Les limites des approches en termes de capital}

Les analyses précédentes nous donnent de solides points de départ pour étudier la dimension temporelle des ressources culturelles. L'analyse beckérienne a le mérite de mettre l'accent sur les effets économiques de la culture, sur la possibilité de la considérer aussi comme ensemble de ressources ayant une valeur économique, sur l'intérêt de se préoccuper de sa gestion efficace en prenant en compte l'ensemble de ses effets économiques. L'approche de la créativité insiste quant à elle sur la spécificité du fonctionnement productif de la culture. Les débats menés autour de ces représentations de la dimension économique de la culture permettent de mettre en évidence quatre spécificités relatives à la dimension temporelle des ressources culturelles. Elles montrent qu'on ne peut se contenter d'identifier les ressources culturelles à du capital, quand bien même elles auraient une dimension et des effets économiques et marchands, dans la mesure où il s'agit d'un capital très spécifique.

\subsubsection{L'absence de soustractibilité temporelle dans l'usage de la ressource}

L'application à la culture de l'approche de la créativité permet de révéler deux caractéristiques centrales des ressources culturelles comme ressources économiques. 
Il n'y a généralement pas de rivalité dans leur usage à travers l'espace parce que leur « consommation » ne les consomme précisément pas et n'interdit pas à d'autres (sous réserve d'effets classiques d'encombrement) d'y recourir. La consommation de programmes télévisés par certains n'empêche pas d'autres de regarder les mêmes programmes et d'en recevoir autant d'utilité. Au contraire, même, nombre de ressources culturelles sont sujettes à des effets de réseaux et leur utilité individuelle s'accroît avec l'extension de leur usage par d'autres (le programme télé vu par de très nombreux téléspectateurs leur permet des échanges plus nombreux que celui qui, à la limite, ne serait vu que par un d'entre eux). Nous sommes ici en présence d'un effet désormais bien connu. En revanche, l'absence de soustractibilité temporelle de ces ressources a suscité moins de curiosité. Pourtant, l'une des caractéristiques essentielles des ressources culturelles est que leur usage dans le temps n'est généralement pas rival. Les lecteurs de La Princesse de Clèves aujourd'hui ne souffrent pas du fait que des générations et des générations les aient précédés dans cette voie. Cette absence de soustractibilité dans le temps s'applique aussi à des rôles conjoints de biens de consommation et biens de production, et, compte tenu de leurs propriétés de non-rivalité, sans que ces fonctions ne s'excluent. La Joconde sera consommée comme bien de consommation par le visiteur amateur mais aussi comme bien de production par le peintre en formation qui l'étudiera et en fera une copie.

\subsubsection{Le profil particulier du coût d'usage du capital culturel}

L'exemple précédent montre que, dans certains cas, un usage préalable du capital culturel renforce sa "productivité " présente. La lecture de La Princesse de Clèves par les générations précédentes permet aux lecteurs contemporains, en les inscrivant dans une culture, de bénéficier des commentaires qu'a suscités la fréquentation passée de cette ressource. La productivité présente du capital culturel peut être accrue par son utilisation passée alors que le capital standard est censé s'user par l'usage et voir sa valeur progressivement décliner. Alors que la consommation des ressources ordinaires est une consommation destructrice, celle des ressources culturelles peut être une consommation valorisatrice. De même, l'obsolescence du capital se présentet-elle dans des conditions différentes. Quand il s'agit de connaissance cumulative, les anciennes ressources sont remplacées par de nouvelles et deviennent obsolètes ; mais, quand il s'agit de connaissance non cumulative, il n'en est pas de même. La peinture de la renaissance italienne, bien que « vieille » de plusieurs siècles, continue à fasciner le public et à façonner le talent de nouveaux peintres.

\subsubsection{Le mode d'action du capital culturel : de la créativité au patrimoine}

L'approche de la créativité limite l'intérêt d'un raisonnement en termes de capital culturel dès lors que celui-ci ne fonctionne pas selon le schéma traditionnel de la fonction de production, c'est-à-dire en établissant une relation régulière et reproductible entre un input clairement désigné et une production de valeur. Si la production se fait en utilisant des patrimoines existants, comment mesurer leur apport ? Comment mesurer (en tenant compte de leur effet dans le temps, en termes de consommation et de production) des « capitaux culturels " que représenteraient Chambord ou Borobudur, KEYNES et RICARDO? Quelle est la "valeur» ou la " productivité » de l'invention de la perspective en peinture, de la création du style New Look dans la mode d'après-guerre, de la mise au point de la cuisine moléculaire ? 
PICASSO explique qu'il n'aurait jamais réalisé son œuvre sans CÉZANNE, mais aussi sans Puvis DE ChAVANne, sans POUSSIN et INGRES, sans VÉLASQUEZ et sans la peinture italienne ; mais comment identifier et séparer les différents inputs, et notamment ceux qui relèvent du patrimoine et ceux qui relèvent de son utilisation créative ${ }^{1}$ ? Quelle part du produit PICASSO attribuer à INGRES et quelle part à CÉZANNE?

\subsubsection{Un capital non générique mais localisé et idiosyncrasique}

L'un des intérêts de la notion de capital est de permettre de comparer l'efficacité de différentes combinaisons productives et de s'aligner sur la combinaison efficiente. Cela suppose qu'on puisse investir en capital pour reproduire une combinaison dont on a pu observer ailleurs l'efficience. Or les ressources culturelles ne sont pas de nature générique. Elles sont généralement incluses dans des ensembles plus vastes eux-mêmes constitués à travers le temps sur la base de conditions historiques et géographiques déterminées et donc impossibles à transposer ailleurs avec le même résultat. S'il est parfois possible d'emprunter à une construction culturelle existante (s'inspirer du toyotisme par exemple pour le transposer en Occident), nombreux sont les cas où cela n'a pas de sens du fait de l'idiosyncrasie des ressources. Cela peut aller jusqu'à la singularité des ressources. Raisonner en termes de ressources créatives permet de penser la singularité des génies mais, plus largement, la singularité de nombre de ressources culturelles. Si l'on peut doubler, tripler ou décupler son parc d'ordinateurs, on ne peut embaucher un, deux ou trois EINSTEIN ou Steve Jobs et on ne peut produire de nouvelles symphonies de MOZART ou de nouveaux tableaux de PICASSO.

Il ne s'agit pas là de spécificités marginales qui permettraient de maintenir l'identification entre ressources culturelles et capital et de les traiter indifféremment comme capital culturel. Le sens du concept de capital en économie est de mettre en relation une dépense (de capital), donc une consommation de valeur avec une production, donc une augmentation de valeur, afin de gérer de façon efficace cette relation via un calcul économique. À partir du moment où l'aspect de dépense ne s'applique plus nécessairement et où celui de production de valeur n'est plus significatif des effets du capital, le concept devient inapproprié. Mieux vaut dire que, dans certaines conditions, les ressources culturelles peuvent fonctionner comme capital, sans d'ailleurs que cela n'épuise nécessairement leurs effets. Dans d'autres, au contraire, elles ne jouent pas selon le schéma du capital. Dès lors, peut-on se tourner vers l'approche des commons qui a pour vocation de traiter de ressources économiques qui ne prennent pas la forme classique de capital mais, au contraire, s'organisent dans des formes institutionnelles collectives?

\subsection{Les ressources culturelles comme cultural commons}

La question des commons concerne en premier lieu les common-pools de ressources naturelles. Après l'article célèbre de HARDIN (1968) on recommanda de choisir entre privatisation et contrôle étatique pour les gérer au lieu de les laisser en libre accès. Néanmoins, le développement des pratiques et des analyses a rapidement montré les limites des politiques de quota ( $c f$. par exemple le cas des langousteries du Maine, WiLSON, 1997) et la possibilité d'une tragédie des anti-commons entraînée par la 
multiplication et le partitionnement des droits de propriété (HELLER, 1998, 2008). OSTROM (1990) a clarifié les choses en distinguant CPR (common-pool resources) et open access et en analysant les différentes formes de propriété commune comme des institutions spécifiques pouvant conduire à des arrangements institutionnels complexes d'organisation de la propriété. Les commons ont été considérés comme un quatrième type de bien dans la classification : biens privés (forte soustractibilité et exclusion facile), biens de club (faible soustractibilité et exclusion facile), biens publics (faible soustractibilité et exclusion difficile) et commons (forte soustractibilité et exclusion difficile). Son étude concernait principalement les communs de ressources naturelles mais cette approche pouvait aussi s'appliquer aux communs culturels (Hess et OSTROM, 2003).

Dans le cas des communs culturels, comme l'utilisation des ressources culturelles n'est pas généralement rivale, le problème est encore plus complexe parce que le critère d'efficience ne peut se limiter à une exploitation évitant gaspillage et surutilisation mais doit concerner leur production et leur extension (MADISON et al., 2010). En outre, de nombreux semi-communs et communs limités, régis par des formes variées de combinaison entre propriété privée, publique et commune, avec des limites à leur ouverture variables, sont présents dans le champ culturel et contribuent fortement aux spill-overs qui caractérisent l'information et la culture ; l'expansion du Web a fait de ce dernier le lieu principal, aujourd'hui, des semi-communs.

La littérature consacrée aux communs culturels et créatifs est particulièrement intéressante. Cependant, elle évolue désormais vers une conception élargie des communs, qui dépasse l'objet originel d'OSTROM (1990), le management des ressources. Celle-ci s'intéressait principalement à la définition des formes institutionnelles capables d'organiser une gestion efficace (définition des ressources, identification des objectifs des communs, définition de l'ouverture, affectation du contrôle, formes de gouvernance et règles d'usage). Comprendre le nouveau rôle des commons dans une économie créative implique de considérer tous leurs effets économiques et sociaux, qu'ils soient directs ou indirects : comment les communs culturels élèvent-ils le capital culturel d'une population, comment développent-ils les motivations intrinsèques pour les activités créatives, favorisent-ils la coopération, créent-ils une atmosphère créative, et ainsi de suite.

Ostrom (1990) étudiait la construction sociale et historique des communs et de leurs règles par des communautés. Néanmoins, son approche de la gestion des ressources demeurait encastrée dans l'approche traditionnelle de l'efficience (même si elle dépassait l'opposition standard entre bien privé et bien public), qu'elle étendait vers la prise en compte des coûts de transaction : pour elle, fréquemment, les communautés étaient de meilleures gestionnaires des coûts de transaction que le marché et l'État. Elle ne développait pas pour autant une approche institutionnaliste, qui étudie explicitement des institutions replacées dans leurs contextes temporel et spatial, au-delà de la seule question de la gestion directe des ressources. Cela se manifestait dans la présentation naturaliste ou techniciste des communs comme quatrième type de bien (forte soustractibilité et exclusion difficile, cf. supra). Les analyses mêmes menées par OsTROM (1990) ont pourtant montré que les formes 
sociales de gestion des communs découlaient aussi de choix stratégiques et de projets, parfois rivaux, nombre de ressources pouvant dans certains cas être gérés de façon privative mais, dans d'autres, via la forme club, la forme publique ou la forme communs.

Une seconde limite, liée à la précédente, est que la construction historique des communs ne crée pas seulement des ressources mais aussi des ressources " négatives ", des habitudes et routines inefficientes, des trends culturels en opposition au progrès et à la créativité. Les communautés héritent du passé, pas seulement des ressources économiques, mais aussi des caractères sociaux qui peuvent avoir des effets économiques et sociaux positifs ou négatifs. Comme NORTH (1991) l'a brillamment montré, ils peuvent développer des effets de lock-in. C'est également dire que certaines ressources (communes mais aussi privatives), comme nous l'avons esquissé, sont particulières en ce qu'elles sont liées à d'autres au sein d'ensembles qui passent à travers le temps et que nous avons appelés des patrimoines. Dès lors, elles ne peuvent être seulement étudiées comme éléments de common-pool resources et leur dimension patrimoniale doit être prise en compte explicitement. D'où l'intérêt de questionner les études portant sur des patrimoines, définis comme des ensembles dont les communautés mais aussi les individus héritent du passé.

\section{-4 - \\ Une approche patrimoniale ?}

Le mouvement de patrimonialisation qui s'est fortement accéléré depuis les années 1980 a suscité l'intérêt de nombreux analystes en sciences sociales. Les économistes, même s'ils ont peu alimenté ces débats, ont tout à gagner à connaître leurs acquis. Nous nous y référerons avant de voir sur quelles bases fonder une analyse économique utilisant la catégorie de patrimoine comme instrument d'analyse de la dimension temporelle des ressources culturelles.

\subsection{Les enseignements des analyses des patrimoines culturels}

\subsubsection{Des précurseurs}

On les trouve dans une tradition institutionnaliste, quelque peu oubliée, initiée en France par DuRKнеiм (1893) pour dépasser l'opposition individualisme - holisme en traitant d'individus socialisés, un courant qui culminera avec l'œuvre de ELIAS (cf. notamment ELIAS, 1973) et la conceptualisation de la société comme société d'individus. À partir de la notion d'interdépendance sociale des individus (que ElIAS (1973) reprendra sous la forme de la société comme réseau symbolisée par l'image du filet de pêche, ensemble de mailles interconnectées) et de l'idée du passage d'une solidarité mécanique ou solidarité par similitude à une solidarité organique liée à la différenciation et l'interdépendance des individus, certains de ses disciples, au premier rang desquels BOUGLÉ (1907) et BOURGEOIS (1902), développeront une approche solidariste - la notion de solidarité étant déjà largement utilisée par DuRKHEIm (1893) -, faisant de la solidarité la forme politique de l'interdépendance 
sociale, conçue comme interdépendance à un moment donné mais aussi, dans le temps, entre générations). Pour eux, chaque individu est né dans une société qui lui permet de bénéficier d'un acquis social et culturel, c'est-à-dire d'un patrimoine. Sans cette relation à la société, personne ne pourrait survivre. Dès lors un contrat implicite (un quasi-contrat pour BOURGEOIS, 1902) lie l'individu et la société : chaque individu a des droits sociaux (droit à l'éducation...) et des devoirs sociaux. On voit comment c'est bien la dimension temporelle des sociétés via celle de sa culture qui, dans cette conception, est à la source de la socialisation des individus.

La dimension temporelle relevée par le Solidarisme se retrouve dans l'école juridique française du Service Public représentée principalement par DuGuit et de l'institution représentée par HAURIOU. Ce dernier, en se fondant sur une théorie de l'État comme institution exprimant une œuvre et donc un projet, s'inscrivant dans la durée, apportera au débat l'idée de puissance de l'État comme condition du maintien de la cohésion du groupe dans le temps. La double dimension de l'institution, à la fois instituée et instituante, conduit également à souligner la prégnance de l'État. Historiquement institué, il institue les individus comme sujets de droit et comme citoyens et non comme échangeurs de biens et services sur des marchés de biens ou de services producteurs. Et, dans ces activités mêmes, leur personnalité juridique contribue à définir le type de contrats, donc de droits et d'obligations, qu'ils sont susceptibles de nouer. Un point de vue analogue est développé par le travaillisme anglais qui, avec MARSHALL (1950), analyse les droits sociaux comme la conséquence du statut de citoyen entendu à la fois comme l'égalité des membres de la communauté par rapport aux droits et aux devoirs et comme l'insertion des individus dans une collectivité, une communauté porteuse d'un patrimoine social commun, patrimoine à dimension politique et culturelle (REISMAN, 2005).

\subsubsection{Les patrimoines culturels}

La conception qui fonde la création en 1790 de la Commission des monuments historiques pour recenser et préserver le patrimoine national et met ainsi en avant pour la première fois dans un texte "officiel » le terme de patrimoine est fondée sur l'idée que le passé lègue au présent des monuments et œuvres remarquables méritant d'être protégés et qu'ils sont désormais la propriété collective de la Nation. Par la suite, l'Angleterre, l'Allemagne, l'Italie et d'autres encore mirent en œuvre à leur tour des politiques du patrimoine. Nombre d'hommes politiques estimèrent que la considération du passé et la préservation de ce qu'il en restait étaient un bon moyen de renforcer la conscience de l'identité nationale à un moment de forte compétition entre nations européennes et d'explosion des nationalismes. Aujourd'hui, les théoriciens de la conservation, conservateurs, anthropologues, archéologues, ont mis en évidence le fait que les patrimoines n'étaient pas les résultats évidents du passé mais procédaient de constructions sociales et culturelles. TUNBRIDGE et ASHWORTH (1996) définissent le patrimoine comme " a contemporary product shaped from history ". C'est une lecture spécifique du passé destinée à ancrer des identités sociales, culturelles et politiques d'individus, de groupes, de lieux. Des conflits se nouent pour les définir, les délimiter et les mettre en forme. Le futurisme italien, souvent proche du mouvement fasciste, mérite-t-il d'être protégé donc valorisé ? Et quid des architectures nazies et staliniennes ? Comment présenter 
les symboles des temps coloniaux, de l'Apartheid ou de l'esclavage ? La corrida doit-elle être considérée comme un élément du patrimoine ibérique ou comme une pratique barbare à oublier?

Le patrimoine prendrait alors un caractère « dissonant » (TUNBRIDGE et ASHWORTH, 1996) parce que la construction et la gestion du patrimoine sont fondées sur une interprétation de l'histoire qui cherche à donner un sens à l'histoire ainsi reconstruite. $\mathrm{C}^{\prime}$ est donc une appropriation de l'histoire qui légitime des groupes et des actes et en exclut d'autres (pour des précisions, $c f$. BARRÈRE, 2014).

Nous sommes ici très loin de l'approche des cultural commons qui les limite à une collection de ressources culturelles, c'est-à-dire de " choses ». Les patrimoines culturels ne sont ni des choses ni des agrégats de choses. Étant liés à des significations ils incorporent une dimension subjective et représentent une relation entre objets et sujets. Comme éléments d'un projet global, ils ne sont pas du genre d'un agrégat mais sont structurés pour définir le sens de leur construction. Cela conduit à distinguer entre ressources culturelles, cultures et patrimoines. Les ressources culturelles, issues de différentes cultures, c'est-à-dire de différentes perceptions des rapports des individus au monde et aux autres dans le cadre de groupes, sont multiples et hétérogènes, à un moment donné. Elles forment une sorte de commonpool de ressources, sans structure ni cohérence particulières. Ainsi les cuisiniers ont-ils à leur disposition une multitude de recettes, issues de multiples cuisines très différentes (française, italienne, chinoise, thaïe, juive...), qui constituent une sorte de " pot commun » dans lequel ils peuvent puiser pour les appliquer ou s'en inspirer pour créer de nouveaux plats ou de nouvelles recettes. Au-delà de la diversité des ressources communes, des traits permettent de distinguer diverses cultures, traditions ou civilisations. Les cultures grecque ou romaine découlent de principes définis qui leur donnent une cohérence et une spécificité, reliées à une identité. Il en est de même pour les principes culinaires qui organisent des cultures culinaires. Les patrimoines, qu'il s'agisse de ceux des groupes, des nations, de l'humanité (le patrimoine commun de l'humanité de l'UNESCO), des firmes ou des individus, résultent d'un travail antérieur de construction (consciente et inconsciente) et d'une accumulation. Ainsi les patrimoines nationaux, issus de cultures déterminées, s'en distinguent en ce qu'ils en cristallisent les éléments identitaires et spécifiques, alors que les cultures peuvent avoir une durée de vie plus courte en ne passant pas l'épreuve de la sélection par l'histoire. Si certaines cultures ou certains éléments culturels seront agglomérés au patrimoine, d'autres pourront disparaître (rien ne dit que la culture hip-hop deviendra un élément du patrimoine national ou local...). Il en est de même pour les patrimoines d'organisations ou d'individus qui résulteront toujours de sélections opérées par le passage du temps irréversible et représenteront les traces et les legs du fonctionnement passé.

\subsection{Analyser la dimension temporelle des ressources culturelles constituées en patrimoines?}

Du point de vue économique, le patrimoine culturel et social, quand on le considère dans le temps, n'est pas seulement une dette sociale de l'individu, comme 
le développe Bourgeors (1902), mais d'abord un actif collectif. Une société produit des marchandises et des institutions. La partie la plus importante de ces biens disparaît dans la consommation, improductive et productive. Certains d'entre eux restent et constituent un patrimoine social et culturel. Le stock de capital est une part importante de ce patrimoine social, mais les biens créatifs (produits artistiques, connaissances...), les règles, les institutions, les normes de comportement sont aussi une autre part essentielle de ce patrimoine. Ils ont des effets forts à la fois sur la consommation et sur la production, sont à la fois inputs et outputs, et les conditions du fonctionnement économique de la société actuelle en dépendent étroitement. La richesse sociale dépend autant du patrimoine social que des choix des agents économiques et, plus l'économie devient économie d'information et de connaissance, plus le rôle de cette source de richesse s'accroît. Réciproquement, la société doit gérer ce patrimoine (tout autant que le patrimoine naturel) pour les générations futures.

Le patrimoine est alors défini comme « ensemble, attaché à un titulaire (un individu ou un groupe, une organisation, une institution, un territoire, bref n'importe quelle "entité") et exprimant sa spécificité, ensemble historiquement institué et territorialement situé d'avoirs construits et transmis par le passé, avoirs qui sont des actifs matériels, des actifs immatériels et des institutions ". Le patrimoine est défini au niveau d'une unité ayant une frontière qui permette de l'autonomiser et de la distinguer des autres unités au sein d'un environnement donné. Les acteurs, individuels et collectifs (les firmes et les territoires notamment), se voient dotés de patrimoines qui contribuent, comme les habitus de BOURDIEU, à leurs prises de décision. Ils peuvent également constituer des ressources spécifiques, atouts compétitifs dans la concurrence, comme nous l'avons vu avec la dialectique créativité-patrimoine. Ces patrimoines, généralement, rassemblent des éléments contradictoires, certains issus de patrimoines culturels marchands, d'autres de patrimoines idéologiques ou socio-politiques (le patrimoine républicain par exemple). Les patrimoines ne sont par nature ni marchands ni non marchands. Même si leurs caractéristiques de valeurs d'usage conditionnent la forme institutionnelle de leur apparition et de leur gestion, celles-ci résultent aussi de constructions sociales, d'inventions, de stratégies et de projets. Dès lors, des patrimoines fonctionnent comme capital, d'autres comme ressources communes et, parfois, les mêmes peuvent fonctionner de façon alternative sur un mode marchand et capitaliste, avec des effets de valeur identifiables, et sur un mode de ressources collectives, voire ils peuvent fonctionner de façon hybride en produisant simultanément des effets marchands et non marchands, individuels et collectifs, appropriés et non appropriés, mesurables et non mesurables. Les biens peuvent à leur tour se voir dotés de propriétés patrimoniales et l'on parlera de biens patrimoniaux pour traiter des marchés du roquefort, du parmesan, du champagne ou de la mode française. Le raisonnement en termes de patrimoine permet de prendre en compte l'ensemble de ces éléments en dépassant la limite précédemment repérée ( cf. $3^{\circ}$, section 2.1 supra) dans la théorie beckérienne du capital culturel et du capital social puisque celle-ci implique de mesurer monétairement les effets en termes de valeur de la productivité de ce capital culturel.

L'approche patrimoniale permet également de tenir compte des effets économiques négatifs que le patrimoine peut engendrer, à la façon dont NORTH (1991) a 
traité des effets incitatifs des logiques institutionnelles à l'œuvre dans le modèle de colonisation marchande appliqué à l'Amérique du Nord et dans le modèle impériobureaucratique imposé en Amérique du Sud et, de façon plus large, des contraintes de sentier. De manière plus générale, elle entre en résonance avec un ensemble de travaux en économie et en sciences sociales mettant en avant les mécanismes de coévolution entre les comportements et les institutions. Ainsi, à partir d'une approche fondée sur l'utilisation de la théorie des jeux évolutionnistes, BOWLES et GINTIS (2011) ont récemment montré comment les institutions (les conventions, les normes, les règles juridiques), par leur effet structurant sur les interactions sociales, sont susceptibles de favoriser l'évolution de certains comportements et de certains profils de préférences dans le temps long. Mais les institutions sont elles-mêmes soumises à un processus d'évolution, généré par les interactions entre les agents au travers de leurs comportements. BOWLES et GINTIS (2011) soulignent ainsi, bien que ce soit avec des outils relevant davantage de l'économie "standard ", le caractère mutuellement constitutif des communautés au sens large et du patrimoine : c'est parce que les interactions sociales des agents s'organisent autour d'un ensemble commun d'institutions que ces derniers sont à même de se coordonner et de coopérer. Simultanément, ces interactions sont constitutives des institutions. La valorisation des biens culturels au sein d'une communauté est une dimension de ce patrimoine. L'approche de BOWLES et GiNTIS (2011) en termes de coévolution institutions/comportements redécouvre d'une certaine manière les analyses des économistes institutionnalistes américains du début du siècle dernier, tels que VEBLEN (1914). Ce dernier est en effet l'auteur méconnu d'une théorie de l'évolution culturelle reposant sur les mêmes mécanismes de coévolution entre les institutions et ce qu'il appelait les « habitudes de pensée et d'agir » (HÉDOIN, 2010).

\section{- 5 - Conclusion}

L'approche patrimoniale permet, on le voit, de s'émanciper des contraintes de l'approche du capital culturel. Elle offre un cadre plus vaste pour apprécier l'ensemble des effets économiques, directs et indirects, de la contrainte représentée par l'irréversibilité du temps et le lien qu'elle instaure entre passé, présent et futur. Elle s'inscrit dans une perspective institutionnaliste, évolutionniste et substantiviste. Substantiviste parce qu'au lieu de rassembler toutes les ressources culturelles sous la dénomination formelle de capital et de les assimiler à tout actif jouant le rôle de capital, elle tient compte de leurs spécificités de valeurs d'usage, en particulier pour comprendre la relation entre leur contenu et leurs formes institutionnelles de gestion et d'appropriation. Institutionnaliste et évolutionniste parce qu'elle resitue les acteurs, les organisations, les institutions et les territoires dans leur contexte social et temporel, dans leur histoire, et dépasse de ce point de vue le cadre statique de l'allocation des ressources utilisé par l'approche des cultural commons en spécifiant acteurs, organisations et institutions. Elle le dépasse aussi en ne se limitant pas au cas des communautés parce que les individus marchands portent eux aussi 
des patrimoines. Enfin, elle intègre la pluralité de patrimoines que peuvent porter individus et communautés.

Si l'appréhension des ressources culturelles comme capital, productif ou créatif, avait le mérite de mettre l'accent sur la dimension économique de ces ressources, sur leur contribution à la richesse et à la production de valeur, elle demeure incapable de prendre en compte la particularité de la forme patrimoniale prise par certaines de ces ressources. La patrimonialisation, explicite ou implicite (par exemple quand les individus se définissent comme porteurs de "personnalités " propres), est une forme d'institutionnalisation des spécificités. Elle développe le caractère idiosyncrasique des ressources qui forment ces patrimoines et les éloigne de ce fait de la généralité que cherche à établir la catégorie de capital. Alors que ce dernier est fondé sur la notion de substituabilité (substituabilité des valeurs d'usage particulières qui constituent des équipements différents, substituabilité du capital au travail) pour obtenir la combinaison productive efficiente, le patrimoine, par sa détermination historique, s'inscrit dans la spécificité et l'idiosyncrasie.

L'approche patrimoniale permet enfin de tenir compte du double aspect du rapport entre culture et marché. Si les ressources et produits culturels sont pour partie happés par les régulations marchande et capitaliste, comme l'annonçait l'École de Francfort, et si, de fait, des patrimoines culturels sont marchandisés et valorisés, les spécificités mêmes des formes culturelles empêchent de transformer toutes ces ressources et produits en simples marchandises ou capitaux. La notion de patrimoine, même si elle a vocation à ne s'appliquer qu'à certains sous-ensembles des ressources culturelles, se situe en amont des notions de capital culturel ou créatif. Ayant un degré de généralité plus grand, elle conduit à analyser la dialectique entre formes privées et collectives, marchandes et non-marchandes, de patrimonialisation et ouvre alors sur une analyse plus riche du lien entre champ culturel et régulation économique.

\section{Références bibliographiques}

BARRÈRE C (2013) Heritage as a basis for creativity in creative industries: the case of taste industries. Mind and Society 12: 167-176.

BARRÈRE C (2014) Les quatre temps du patrimoine, Économie Appliquée (à paraître).

BARRÈre C, SANTAGATA W (1999) Defining art: from the Brancusi trial to the economics of artistic semiotic goods, International Journal of Arts Management 1 (2): 28-40.

Barrère C, Barthélémy D, Nieddu M, Vivien F-D (dir.) (2004) Réinventer le patrimoine. L'Harmattan, Paris. BECATTINI G (1998), Distretti industriali e made in Italy. Le basi socioculturali del nostro sviluppo economico. Bollati Boringhieri, Torino.

BECKER G (1964) Human capital: a theoretical and empirical analaysis, with special reference to education. University of Chicago Press, Chicago.

BECKER G (1996) Accounting for tastes. Harvard University Press, Cambridge.

BENJAMIN W (1936) The work of art in the age of mechanical reproduction. [en ligne] http://www.marxists.org/reference/subject/philosophy/works/ge/benjamin.htm.

Bertacchini E, Bravo G, Marrelli M, Santagata W (2012) Cultural commons, a new perspective on the production and evolution of cultures. Edward Elgar, London. 
BOUGLÈ C (1907) Le solidarisme. Giard et Brière, Paris.

Bourgeols L $(1902,1996)$ Solidarité. Presses du Septentrion, Toulouse.

BOWLES S, GINTIS H (2011) A cooperative species. Princeton University Press, Princeton and Oxford.

CAVES RE (2000) Creative industries. Contracts between art and commerce. Harvard University Press, Cambridge.

DCMS (2001) UK Government Department for Culture, Media and Sport. Annual Report 2001. [en ligne] www.culture.gov.uk/. Accès : 12 juin 2012.

DURKHEIM E (1893) De la division du travail social. PUF, Paris.

ELIAS N (1973) La civilisation des mœurs. Calmann-Lévy, Paris.

FLORIDA R (2002) The rise of the creative class. and how it's transforming work, leisure and everyday life. Basic Books, New York.

HARDIN G (1968) The Tragedy of the commons. Science 162: 1243-1248.

HÉDOIN C (2010) Did veblen generalize darwinism (and why does it matter)? Journal of Economic Issues 44(4): 963-990.

HeLleR M (1998) The Tragedy of the anticommons. Harvard Law Review (1).

HeLLER M (2008) The gridlock economy: how too much ownership wrecks markets, stops innovation, and costs lives. Basic Books, New York.

HesS C, OStROM E (2003) Ideas, artifacts, and facilities: information as a common-pool resource. Law and Contemporary Problems 66: 111-145.

HORKHEIMER M, ADORNO TW (1947) Dialectic of enlightment. philosophical fragments. English translation (2002). Gunzelin Schmid Noerr, Frankfurt.

KEA (2006) The economy of culture in Europe. Study prepared for the European Commission (DirectorateGeneral for Education and Culture). [en ligne] www.keanet.eu/ecoculture/studynew.pdf. Accès : 14 juin 2012.

LIPOVETSKY G (1987) L'empire de l'éphémère. Gallimard, Paris.

MADISON M J, FrISChMANN B M, StRANDBURG K J (2010) Constructing cultural commons in the cultural environment. Cornell Law Review 95: 657-710.

MARSHALL TH (1950) Citizenship and social class and other essays, Cambridge University Press, Cambridge.

Ministère Des Affaires Étrangères et EuropéENnes (2009) Rapport Industries culturelles. [en ligne] www.diplomatie.gouv.fr/.../091005. Accès : 14 juin 2012.

NORTH D (1991) Institutions, institutional change and economic performance. Cambridge University Press, Cambridge.

OStROM E (2007) Governing the commons: the evolution of institutions for collective action. Cambridge University Press, Cambridge.

POTTS J (2012) Creative industries and economic evolution. Edward Elgar, Cheltenham.

SANTAGATA W (2002) Cultural districts, property rights and sustainable economic growth. International Journal of Urban and Regional Research, 26 (1): 9-23.

StEINER G (2001) Grammaires de la création. Gallimard, Paris.

THROSBY D (1999) Cultural capital. Journal of Cultural Economics 23(1): 3-12.

THROSBY D (2001) Economics and culture, Cambridge University Press, Cambridge.

TOWSE R (2003) A handbook of cultural economics. Edward Elgar, Cheltenham.

TUNBRIDGE J E, ASHWORTH G J (1996) Dissonant heritage: the management of the past as a resource in conflict. Wiley, Hoboken.

VEBLEN T $(1914,1990)$ The Instinct of workmanship and the state of the industrial arts, B.W. Huebsch, New York, Trasaction Publishers, New Jersey.

WILSON JA (1997) Maine's lobster fishery. Managing a common property resource, Internet.

ZUKIN S, BRASLOW L (2011) City, culture and society. Elsevier, London, Amsterdam. 
Du capital culturel aux patrimoines culturels

\section{Notes}

1 - L'additivité de la fonction de production disparaît parce que chaque élément du processus créatif et chaque intervention d'effets patrimoniaux peut jouer un rôle décisif, indépendamment de son coût absolu et relatif, comme l'exprime la théorie de la production O-ring (c'est la défaillance d'un joint $\mathrm{O}$, pièce de valeur insignifiante, qui aurait suffi à causer l'explosion au décollage de la navette Challenger). 